\title{
Chiral Logs in the Presence of Staggered Flavor Symmetry Breaking
}

\author{
C. Bernard \\ (MILC Collaboration) \\ Washington University, St. Louis, Missouri 63130, USA
}

(October 24, 2018)

\begin{abstract}
Chiral logarithms in $m_{\pi}^{2}$ are calculated at one loop, taking into account the leading contributions to flavor symmetry breaking due to staggered fermions. I treat both the full QCD case (2+1 light dynamical flavors) and the quenched case; finite volume corrections are included. My starting point is the effective chiral Lagrangian introduced by Lee and Sharpe. It is necessary to understand the one-loop diagrams in the "quark flow" picture in order to adjust the calculation to correspond to the desired number of dynamical quarks.
\end{abstract}

PACS numbers: 12.39.Fe, 12.38.Gc, 11.30.Rd

\section{INTRODUCTION}

Staggered (Kogut-Susskind, KS) fermions provide a competitive method for simulating full QCD, including the effects of virtual $u, d$ and $s$ pairs. Indeed, recent improvements in the action [1] have made it possible to compute many physical quantities with rather small scaling violations in such "2+1 flavor" simulations [2,3].

The violation of KS-flavor symmetry, while reduced by the improved ("Asqtad") action, remains however quite large at the lattice spacing $(a \approx 0.13 \mathrm{fm})$ where most of the simulations in Refs. [2,3] have been performed. The maximum splitting in mass squared among the various flavor pions, $\Delta_{\max }$, is $\approx(400 \mathrm{MeV})^{2}$ at this lattice spacing. Because one can choose the staggered flavor on the valence quark lines, the flavor violations often enter through loop effects alone, and as such they have a typical size of only $\sim 8 \%$ in most quantities. Here, I 
estimate the size from a "typical" chiral logarithm, including flavor violation:

$$
\frac{\Delta_{\max }}{16 \pi^{2} f_{\pi}^{2}} \ln \left(\Delta_{\max } / \Lambda^{2}\right),
$$

where $\Lambda$ is the chiral scale (taken, for instance equal to $m_{\rho} \approx 770 \mathrm{MeV}$ ), and $f_{\pi} \cong 131 \mathrm{MeV}$. Since the leading flavor violating terms in the improved KS action are $\mathcal{O}\left(a^{2}\right)$, these discretization effects can be reduced still more by going closer to the continuum limit; MILC simulations are currently in progress at $a \approx 0.09 \mathrm{fm}$.

On the other hand, if one focuses directly on chiral logs and works at $a \approx 0.13 \mathrm{fm}$, the effect of KS-flavor violation should be large. Indeed, in Ref. [3], we were unable to fit $m_{\pi}^{2} / m_{\ell}$ to the standard continuum chiral-log form [4] for $2+1$ flavor QCD. (Here and below $\ell$ stands for a generic light ( $u$ or $d$ ) quark; I neglect isospin violations throughout.) To test the hypothesis that KS-flavor violations are responsible for this behavior of $m_{\pi}^{2} / m_{\ell}$, one needs to compute the chiral logs in the presence of KS symmetry breaking. That computation is the subject of this paper [5].

The effective chiral Lagrangian that describes KS fermions through $\mathcal{O}\left(m, a^{2}\right)$ ( $m$ is a generic quark mass) has been constructed by Lee and Sharpe [6]. Their approach is to make a joint expansion in $m$ and $a^{2}$, which are considered to be comparably small. That is in fact the case for a simulation like [3], where the splittings in mass squared of various KS-flavor pions are comparable to the squared masses themselves. A one-loop calculations using the Lagrangian of Ref. [6] would give the chiral logs (non-analytic terms) of $\mathcal{O}\left(\mathrm{m}^{2}, m a^{2}, a^{4}\right)$.

The Lee-Sharpe Lagrangian corresponds to a single lattice KS field, which becomes 4 degenerate flavors in the continuum limit. The main subtleties in the current work arise because I am interested in the more phenomenologically relevant $2+1$ flavor theory being simulated, with $m_{s} \neq m_{\ell}$ in general, not the 4-flavor (continuum degenerate) KS theory. I follow a three-step procedure:

1. Generalize (almost trivially) the Lee-Sharpe Lagrangian to the $4+4$ case, where one has two lattice KS fields with different masses $\left(m_{\ell}, m_{s}\right)$. This is an $S U(8)_{L} \times S U(8)_{R}$ theory, with mass terms and $\mathcal{O}\left(a^{2}\right) \mathrm{KS}$-flavor violating terms breaking the symmetry. 
2. Compute $m_{\pi}^{2}$ at one loop in the $4+4$ case. I treat only the case where the pion is the $U(1)_{A}$ Goldstone particle. (This is the situation most immediately relevant to the fits attempted in [3].) The symmetry then implies that $m_{\pi}^{2}$ vanishes when $m_{\ell} \rightarrow 0$, allowing one to simplify the calculation.

3. Adjust the $4+4$ answer to correspond to the $2+1$ case of interest. This requires identifying the contributions that correspond to $\ell$ or $s$ virtual quark loops and multiplying them by $1 / 2$ or $1 / 4$ respectively.

The subtleties are almost entirely in step 3. To identify those terms that should be reduced by a factor of 2 or 4, one needs to follow the "quark flow" approach, which was introduced by Sharpe [7] to compute quenched chiral logarithms. Unfortunately, in the presence of KS-flavor symmetry violation, there does not appear to be an alternative, Lagrangian approach [8,9] that would automatically cancel the effects of the unwanted KS flavors. This corresponds to the fact that, in numerical simulations, the reduction from $4+4$ to $2+1$ flavors is accomplished by taking the square root and fourth root of the $\ell$ and $s$ determinants, respectively. The procedure reduces each of the four KS flavors in a virtual quark loop equally, by a factor of 2 (4); it does not than cancel specific flavors. There is thus no equivalent (ultra)local lattice Lagrangian, and it is not at all clear how or whether one can represent the low energy chiral properties of this theory with an effective chiral Lagrangian [10].

Of course, in the continuum limit the KS-flavor symmetry is restored, and one may cancel any 2 of the 4 light quark flavors (and any 3 of the 4 strange quark flavors) with 2 (3) bosonic pseudo-quarks. The effective chiral theory is then obvious: an "8|5" partially quenched chiral perturbation theory. This is discussed in Ref. [9]. Such a theory is trivially equivalent to standard 3 flavor chiral perturbation theory in the physical sector where the sea quarks are also the ones on the external lines.

The remainder of this paper is organized as follows: Section [1] presents the Lee-Sharpe Lagrangian and generalizes it to the $4+4$ case. The calculation of $m_{\pi}^{2}$ to one loop is then 
described in Sec. III. In Sec. IV, the quark flow picture is applied to compute $m_{\pi}^{2}$ in the $2+1$ case. The analytic terms at $\mathcal{O}\left(m^{2}, m a^{2}, a^{4}\right)$, necessary to perform consistent fits that include the chiral logs, are also touched upon. The quenched case follows in Sec. $\nabla$. Finite volume corrections are then discussed briefly in Sec. VI. Finally, I make some remarks about the extension of the current approach to other physical quantities in Sec. VII. That section also contains some comments about the fits of MILC data to the chiral forms derived here; however, a detailed discussion of such fits and their consequences will be presented elsewhere [11].

\section{LEE-SHARPE LAGRANGIAN FOR 4+4 FLAVORS}

Lee and Sharpe [6] first construct the continuum effective Lagrangian for KS lattice fermions, including all terms at $\mathcal{O}\left(a^{2}\right)$. The KS-flavor symmetry is broken by 6-quark operators. They then write down the corresponding effective chiral theory to leading order in $a^{2}$ and $m$, the quark mass.

For a single KS field (4 continuum flavors) the Lee-Sharpe chiral Lagrangian describing the pseudo-Goldstone bosons has a (nonlinear) $S U(4)_{L} \times S U(4)_{R}$ symmetry, broken by the mass term and by the KS-flavor violating operators. The $4 \times 4$ matrix $\Sigma$ is defined by

$$
\Sigma(x) \equiv \exp (i \phi / f), \quad \phi \equiv \sum_{a=1}^{16} \phi_{a} T_{a}
$$

where $\phi_{a}$ are real, $f$ is the tree-level pion decay constant (I use the normalization $f_{\pi} \cong$ $131 \mathrm{MeV})$, and the Hermitian generators $T_{a}$ are chosen as follows,

$$
T_{a}=\left\{\xi_{5}, i \xi_{\mu 5}, i \xi_{\mu \nu}, \xi_{\mu}, I\right\}
$$

Here $\xi_{\mu}$ are the flavor gamma matrices, $\xi_{\mu 5} \equiv \xi_{\mu} \xi_{5}, \xi_{\mu \nu} \equiv \xi_{\mu} \xi_{\nu}$ (with $\mu<\nu$ in eq. (3)), and $I$ is the $4 \times 4$ identity matrix. $\Sigma$ transforms by $\Sigma \rightarrow L \Sigma R^{\dagger}$ under $S U(4)_{L} \times S U(4)_{R}$.

Note that I include the singlet meson $\phi_{I}$, corresponding to generator $I$, in eqs. (2) and (3). The mass of the $\eta^{\prime}$-like meson (often called $\Phi_{0}$, up to overall normalization) gets a large 
contribution $\left(\equiv m_{0}\right)$ from the anomaly in the Lee-Sharpe case and is not included in their formalism. But I want to generalize to two KS fields (8 continuum flavors). In that case, only the $S U(8)$ flavor singlet is heavy and can be integrated out. $S U(4)$ singlets, e.g., a singlet "kaon" made of one quark from each KS multiplet, can be light. I therefore leave the singlet as well as the $m_{0}$ term explicit in intermediate steps, but will ultimately take $m_{0} \rightarrow \infty$.

The (Euclidean) Lee-Sharpe Lagrangian is then

$$
\mathcal{L}_{(4)}=\frac{f^{2}}{8} \operatorname{tr}\left(\partial_{\mu} \Sigma \partial_{\mu} \Sigma^{\dagger}\right)-\frac{1}{4} \mu m f^{2} \operatorname{tr}\left(\Sigma+\Sigma^{\dagger}\right)+\frac{2 m_{0}^{2}}{3}\left(\phi_{I}\right)^{2}+a^{2} \mathcal{V}
$$

where $\mu$ is a constant with units of mass, the coefficient of $\phi_{I}$ is conventional, 2 and $\mathcal{V}$ is the lowest order KS-flavor breaking potential:

$$
\begin{aligned}
-\mathcal{V}= & C_{1} \operatorname{tr}\left(\xi_{5} \Sigma \xi_{5} \Sigma^{\dagger}\right) \\
& +C_{2} \frac{1}{2}\left[\operatorname{tr}\left(\Sigma^{2}\right)-\operatorname{tr}\left(\xi_{5} \Sigma \xi_{5} \Sigma\right)+\text { h.c. }\right] \\
& +C_{3} \frac{1}{2} \sum_{\nu}\left[\operatorname{tr}\left(\xi_{\nu} \Sigma \xi_{\nu} \Sigma\right)+h . c .\right] \\
& +C_{4} \frac{1}{2} \sum_{\nu}\left[\operatorname{tr}\left(\xi_{\nu 5} \Sigma \xi_{5 \nu} \Sigma\right)+h . c .\right] \\
& +C_{5} \frac{1}{2} \sum_{\nu}\left[\operatorname{tr}\left(\xi_{\nu} \Sigma \xi_{\nu} \Sigma^{\dagger}\right)-\operatorname{tr}\left(\xi_{\nu 5} \Sigma \xi_{5 \nu} \Sigma^{\dagger}\right)\right] \\
& +C_{6} \sum_{\mu<\nu}\left[\operatorname{tr}\left(\xi_{\mu \nu} \Sigma \xi_{\nu \mu} \Sigma^{\dagger}\right) .\right.
\end{aligned}
$$

\footnotetext{
${ }^{1}$ Sharpe and Shoresh [12] show that integrating out the $\Phi_{0}$ is mathematically equivalent to keeping it in the calculations and taking the mass parameter $m_{0} \rightarrow \infty$ at the end. If that limit is to be taken, it is unnecessary to include $\Phi_{0}$ dependence other than the $m_{0}$ term in the action. However, in the quenched case (Sec. $\mathrm{V}$ ), other $\Phi_{0}$ dependence will need to be considered.

${ }^{2} m_{0}^{2} / 6$ by definition multiplies $\left(\phi_{11}+\phi_{22}+\phi_{33}+\cdots\right)^{2}$. Here, the normalized $\phi_{I} \equiv \frac{1}{2} \sum_{i=1}^{4} \phi_{i i}$. This definition of $m_{0}^{2}$ is independent of the number of flavors and corresponds to that in Refs. [8,9] (after renaming $\mu^{2} \rightarrow m_{0}^{2}$ ) and that in Ref. [7] (after choosing $N=3$ there). With the current definition of $f$, the parameter $\delta$ introduced in $[7]$ is equal to $m_{0}^{2} /\left(24 \pi^{2} f^{2}\right)$.
} 
Effects of order $a^{2} m$ or $a^{4}$ are neglected in eq. (41).

The potential $\mathcal{V}$ has an "accidental" $S O(4)$ flavor symmetry (evidenced by the sums over $\nu$ and $\mu$ ), which is larger than the lattice symmetry group. The pions fall into $5 S O(4)$ representations with flavors $\xi_{5}, \xi_{\mu 5}, \xi_{\mu \nu}, \xi_{\mu}, I$. Expanding eq. (雨 to quadratic order in $\phi$, the pion masses are found to be:

$$
m_{\pi_{B}}^{2}=2 \mu m+\frac{4 m_{0}^{2}}{3} \delta_{B, I}+a^{2} \Delta\left(\xi_{B}\right)
$$

where, in a convenient abuse of notation that will be used from now on, the index $B$ takes the 16 values $\{5, \mu 5, \mu \nu(\mu<\nu), \mu, I\}$ and $\xi_{I} \equiv I$. The $m_{0}^{2}$ contribution to the mass of $\phi_{I}$ is shown explicitly, and $\Delta\left(\xi_{B}\right)$ is given by

$$
\begin{aligned}
\Delta\left(\xi_{5}\right) & =0 \\
\Delta\left(\xi_{\mu 5}\right) & =\frac{16}{f^{2}}\left(C_{1}+C_{2}+3 C_{3}+C_{4}-C_{5}+3 C_{6}\right) \\
\Delta\left(\xi_{\mu \nu}\right) & =\frac{16}{f^{2}}\left(2 C_{3}+2 C_{4}+4 C_{6}\right) \\
\Delta\left(\xi_{\mu}\right) & =\frac{16}{f^{2}}\left(C_{1}+C_{2}+C_{3}+3 C_{4}+C_{5}+3 C_{6}\right) \\
\Delta(I) & =\frac{16}{f^{2}}\left(4 C_{3}+4 C_{4}\right)
\end{aligned}
$$

$\Delta\left(\xi_{5}\right)$ vanishes because of the staggered-flavor nonsinglet $U_{A}(1)$, which is represented in the chiral theory by

$$
\Sigma \rightarrow e^{i \theta \xi_{5}} \Sigma e^{i \theta \xi_{5}}
$$

with $\theta$ the $U_{A}(1)$ angle. Since this symmetry is unbroken by the lattice regulator, $\pi_{5}$ is a true Goldstone boson in the chiral limit.

From simulations such as those in Ref. [3], one learns that the $C_{4}$ term in eq. (5) is the largest contributor to the $\mathcal{O}\left(a^{2}\right)$ flavor violation [6]. This leads to approximately equal splitting between the pions, in the order $\pi_{5}, \pi_{\mu 5}, \pi_{\mu \nu}, \pi_{\mu}, \pi_{I}$.

\footnotetext{
${ }^{3}$ Omitting the disconnected terms in the $\pi_{I}$ propagator (as is often done in simulations - see,
} 
entirely absent, however; their contributions to the splittings are of order $10 \%$ of that of $C_{4}$ [11]. The reason for the smallness of $C_{i}, i \neq 4$, is not known.f

We now need to generalize to the case of two KS fields with different masses $m_{\ell}$ and $m_{s}$, i.e., $4+4$ continuum flavors. The field $\Sigma$ in eq. (2) becomes an $8 \times 8$ matrix, given by

$$
\Sigma(x) \equiv \exp (i \Phi / f), \quad \Phi \equiv\left(\begin{array}{cc}
\pi & K \\
K^{\dagger} & S
\end{array}\right),
$$

where the $4 \times 4$ fields $\pi, K$, and $S$ describe "pions," "kaons," and " $s \vec{s}$ " mesons, respectively. As in eqns. (2, 3),$\pi \equiv \sum_{a=1}^{16} \pi_{a} T_{a}$, with $\pi_{a}$ real, and similarly for $K$ and $S$, except that the $K_{a}$ are complex. The $S U(8)$ singlet $\propto \operatorname{tr} \pi+\operatorname{tr} S$ will be eliminated below by taking the $m_{0} \rightarrow \infty$ limit.

Defining the $8 \times 8$ mass matrix $\mathcal{M}$,

$$
\mathcal{M} \equiv\left(\begin{array}{cc}
m_{\ell} I & 0 \\
0 & m_{s} I
\end{array}\right)
$$

the $S U(8)_{L} \times S U(8)_{R}$ Lagrangian that generalizes $\mathcal{L}_{(4)}$ of eq. (4) is then

$$
\mathcal{L}_{(4+4)}=\frac{f^{2}}{8} \operatorname{tr}\left(\partial_{\mu} \Sigma \partial_{\mu} \Sigma^{\dagger}\right)-\frac{1}{4} \mu f^{2} \operatorname{tr}\left(\mathcal{M} \Sigma+\mathcal{M} \Sigma^{\dagger}\right)+\frac{2 m_{0}^{2}}{3}\left(\pi_{I}+S_{I}\right)^{2}+a^{2} \mathcal{V}
$$

Generalization of the KS-flavor breaking potential $\mathcal{V}$ in eq. (5) requires a little thought. For a single KS field, the symmetry breaking 4-quark operators in the effective continuum theory have the generic form

$$
\bar{Q}\left(\gamma_{S} \otimes \xi_{F}\right) Q \bar{Q}\left(\gamma_{S^{\prime}} \otimes \xi_{F^{\prime}}\right) Q
$$

where $\gamma_{S}$ is an arbitrary spin matrix, $\xi_{F}$ is an arbitrary flavor matrix, and the indices $S, S^{\prime}, F, F^{\prime}$ are contracted in various ways determined by the lattice symmetries. The effective chiral operators may then be found from the 4-quark operators by treating $\xi_{F}$ and $\xi_{F^{\prime}}$ as spurion fields [6].

e.g., [3]), eliminates the $m_{0}$ contribution to the singlet mass and extends the approximately equal splitting rule to KS flavor $I$.

${ }^{4}$ More precisely, the approximately equal splittings imply only that $C_{6}, C_{1}+C_{2}$, and $2 C_{3}-C_{5}$ are small. I thank M. Golterman for pointing this out. 
With two KS fields on the lattice, there is an exact vector $S U(2)$ (broken only by $m_{\ell} \neq$ $\left.m_{s}\right)$ that mixes them. The symmetry guarantees that the KS-flavor breaking 4-quark operators now have either of the two forms:

$$
\bar{Q}_{i}\left(\gamma_{S} \otimes \xi_{F}\right) Q_{j} \bar{Q}_{j}\left(\gamma_{S^{\prime}} \otimes \xi_{F^{\prime}}\right) Q_{i}, \quad \bar{Q}_{i}\left(\gamma_{S} \otimes \xi_{F}\right) Q_{i} \bar{Q}_{j}\left(\gamma_{S^{\prime}} \otimes \xi_{F^{\prime}}\right) Q_{j}
$$

where $i$ and $j(=1,2)$ are $S U(2)$ indices. The operators in eq. (13) are "flavor mixed" and "flavor unmixed," respectively. By Fierz transformation, the flavor mixed operators can be brought to the flavor unmixed form, so we may assume all 4-quark operators are of the latter type. 5 But if all 4-quark operators are flavor unmixed, then all spurion fields are $S U(2)$ singlets. In other words, for $\mathcal{V}$ in eq. (11) we may take simply eq. (5) with the replacement

$$
\xi_{B} \rightarrow\left(\begin{array}{cc}
\xi_{B} & 0 \\
0 & \xi_{B}
\end{array}\right) .
$$

The tree level masses of the pions, kaons, and $S(s \bar{s})$ mesons are then

$$
\begin{aligned}
& m_{\pi_{B}}^{2}=2 \mu m_{\ell}+a^{2} \Delta\left(\xi_{B}\right) \\
& m_{K_{B}}^{2}=\mu\left(m_{\ell}+m_{s}\right)+a^{2} \Delta\left(\xi_{B}\right) \\
& m_{S_{B}}^{2}=2 \mu m_{s}+a^{2} \Delta\left(\xi_{B}\right),
\end{aligned}
$$

where $\Delta\left(\xi_{B}\right)$ are given by eq. (7), and the $m_{0}$ terms are not included because we will treat $2 m_{0}^{2}\left(\pi_{I}+S_{I}\right)^{2} / 3$ in eq. (11) as a vertex (summed to all orders) below.

\footnotetext{
${ }^{5}$ There are also corresponding axial symmetries, whose generators are the direct product of the $U_{A}(1)$ generator, $\gamma_{5} \otimes \xi_{5}$, with the vector $S U(2)$ generators, but I will not need them here.

${ }^{6}$ This may be at the expense of mixing the color indices (suppressed in eqs. (12) and (13)), but they are irrelevant in the corresponding chiral effective theory because of confinement.
} 


\section{ONE-LOOP PION MASS FOR 4+4 DYNAMICAL FLAVORS}

The calculation of $m_{\pi}^{2}$ to one loop in the $4+4$ theory (eq. (11)) is now straightforward. I confine my attention to corrections to the Goldstone pion mass $m_{\pi_{5}}$. The cases of the non-Goldstone pions and the kaons, which will also be useful in confronting simulation data, will be left to future publications.

The graphs are all tadpoles, with vertices coming from the kinetic energy term $\operatorname{tr}\left(\partial_{\mu} \Sigma \partial_{\mu} \Sigma^{\dagger}\right)$, the mass term $\operatorname{tr}\left(\mathcal{M} \Sigma+\mathcal{M} \Sigma^{\dagger}\right)$, and the symmetry violating term $\mathcal{V}$. They are shown in Figs. 1, 2, and 3, respectively.

I write the one-loop $\pi_{5}$ self energy as]

$$
\Sigma\left(p^{2}\right)=\frac{1}{96 \pi^{2} f^{2}}\left(p^{2} \Sigma_{1}+\Sigma_{2}^{\text {con }}+\Sigma_{2}^{\text {disc }}\right)
$$

where I have explicitly separated the contribution coming a disconnected propagator in the internal loop, $\Sigma_{2}^{\text {disc }}$ (corresponding to Fig. 2(b)), from the other, connected contributions in $\Sigma_{2}^{\text {con }}$. The terms "connected" and "disconnected" are applicable at the QCD level: the disconnected diagrams contain gluon intermediate states in the meson loop (Fig. 屯(d),(e),(f)). The $U_{A}(1)$ symmetry guarantees that the sum of $\Sigma_{2}^{\text {con }}$ and $\Sigma_{2}^{\text {disc }}$ is proportional to $m_{\pi_{5}}^{2}$. However, this is not true for individual diagrams contributing to $\Sigma_{2}^{\text {con }}$, so I do not include the $m_{\pi_{5}}^{2}$ factor in the definition.

For the moment, I calculate only the universal chiral logarithms, and ignore the analytic terms in the diagrams (which means the divergences are also ignored). The logarithms come from the following integrals:

$$
\begin{aligned}
& \mathcal{I}_{1} \equiv \int \frac{d^{4} q}{(2 \pi)^{4}} \frac{1}{q^{2}+m^{2}} \rightarrow \frac{1}{16 \pi^{2}} m^{2} \ln m^{2} \\
& \mathcal{I}_{2} \equiv \int \frac{d^{4} q}{(2 \pi)^{4}} \frac{q^{2}}{q^{2}+m^{2}}=-m^{2} \mathcal{I}_{1}+\int \frac{d^{4} q}{(2 \pi)^{4}} \rightarrow-\frac{1}{16 \pi^{2}} m^{4} \ln m^{2}
\end{aligned}
$$

\footnotetext{
${ }^{7}$ The distinction between the field matrix $\Sigma$ in the Lagrangian and the self energy $\Sigma-$ both conventional notations - should be clear from context.
} 
$\Sigma_{1}$ gets contributions only from Fig. 1(a). The result is

$$
\Sigma_{1} \rightarrow-8 m_{\pi_{\mu 5}}^{2} \ln m_{\pi_{\mu 5}}^{2}-8 m_{\pi_{\mu}}^{2} \ln m_{\pi_{\mu}}^{2}-\sum_{B} m_{K_{B}}^{2} \ln m_{K_{B}}^{2}
$$

where no sum on $\mu$ is implied, $B$ takes the 16 values $\{5, \mu 5, \mu \nu(\mu<\nu), \mu, I\}$ as usual, and the arrow means that only the chiral logarithms are included, as in eqs. (17) and (18). Figure [1(b) contributes only to $\Sigma_{2}^{\text {con: }}$

$$
\Sigma_{2, \mathrm{KE}}^{\mathrm{con}} \rightarrow 8 m_{\pi_{\mu 5}}^{4} \ln m_{\pi_{\mu 5}}^{2}+8 m_{\pi_{\mu}}^{4} \ln m_{\pi_{\mu}}^{2}+\sum_{B} m_{K_{B}}^{4} \ln m_{K_{B}}^{2}
$$

again with no sum on $\mu$. Note that among the pions, only the vector and axial vector flavors contribute in eqs. (19) and (20). It is easy to show, using the fact that $\left[\xi_{5}, I\right]=\left[\xi_{5}, \xi_{\mu \nu}\right]=$ $\left[\xi_{5}, \xi_{5}\right]=0$, that no terms of the form $\pi_{5}^{4}, \pi_{5}^{2} \pi_{\mu \nu}^{2}$, or $\pi_{5}^{2} \pi_{I}^{2}$ can be generated by the kinetic energy term. The absence of coupling to $\pi_{I}^{2}$ explains why the kinetic energy term does not contribute to $\Sigma_{2}^{\text {disc }}$.

Figure 2(a) contributes only to $\Sigma_{2}^{\text {con }}$. Couplings of $\pi_{5}$ to all the other pions are generated by the mass vertex, so there is no restriction on the terms that can enter. I find

$$
\begin{array}{rlr}
\Sigma_{2, \mathrm{MASS}}^{\mathrm{con}} \rightarrow & -3 m_{\pi_{5}}^{4} \ln m_{\pi_{5}}^{2}-4 m_{\pi_{5}}^{2} m_{\pi_{\mu 5}}^{2} \ln m_{\pi_{\mu 5}}^{2} \\
& -18 m_{\pi_{5}}^{2} m_{\pi_{\mu \nu}}^{2} \ln m_{\pi_{\mu \nu}}^{2}-4 m_{\pi_{5}}^{2} m_{\pi_{\mu}}^{2} \ln m_{\pi_{\mu}}^{2} & \\
& -3 m_{\pi_{5}}^{2} m_{\pi_{I}}^{2} \ln m_{\pi_{I}}^{2}-\left(m_{\pi_{5}}^{2}+m_{K_{5}}^{2}\right) \sum_{B} m_{K_{B}}^{2} \ln m_{K_{B}}^{2} .
\end{array}
$$

Here, the factors of $m_{\pi_{5}}^{2}$ or $m_{K_{5}}^{2}$ in each term come from the quark masses, which are proportional to Goldstone particle masses squared by eq. (15) with $\Delta\left(\xi_{5}\right)=0$ (eq. (7)) .

The mass vertex also generates the only contributions to the disconnected diagram, Fig. 2(b). This is the sum of $1,2,3, \cdots$ insertions of the vertex $\frac{2 m_{0}^{2}}{3}\left(\pi_{I}+S_{I}\right)^{2}$ on the internal $\pi_{I}$ line. The $\pi_{I}$ line in turn comes from a term proportional to $\pi_{5}^{2} \pi_{I}^{2}$ in the mass vertex; the same term generates the connected contribution $-3 m_{\pi_{5}}^{2} m_{\pi_{I}}^{2} \ln \left(m_{\pi_{I}}^{2}\right)$ in eq. (21). A single insertion of the $m_{0}^{2}$ vertex, which would be the only contribution in the quenched case, gives

$$
\Sigma_{2}^{\text {disc,quench }}=3 m_{\pi_{5}}^{2} \int \frac{d^{4} q}{\pi^{2}} \frac{4 m_{0}^{2} / 3}{\left(q^{2}+m_{\pi_{I}}^{2}\right)^{2}} .
$$


The standard quenched double pole is evident. Iterating the $m_{0}^{2}$ vertex and summing the geometric series then results in

$$
\Sigma_{2}^{\text {disc }}=3 m_{\pi_{5}}^{2} \int \frac{d^{4} q}{\pi^{2}} \frac{4 m_{0}^{2} / 3}{\left(q^{2}+m_{\pi_{I}}^{2}\right)^{2}\left(1+\frac{4 m_{0}^{2} / 3}{q^{2}+m_{\pi_{I}}^{2}}+\frac{4 m_{0}^{2} / 3}{q^{2}+m_{S_{I}}^{2}}\right)}
$$

There is no here need to take the $m_{0}^{2} \rightarrow \infty$ limit or to perform the integral; the conversion to the $2+1$ flavor case can best be done directly from this expression. Note that $\pi_{I}$ and $S_{I}$ intermediate states now enter. As discussed in Sec. [V], these correspond to virtual $\ell$ and $s$ quark loop contributions.

Finally, we need to consider the KS-flavor violating vertices, eq. (5). It is not hard to show that, as in the kinetic energy case, only the vector and axial pions contribute. From Fig. 3, I obtain (no sum on $\mu$ )

$$
\Sigma_{2, \mathcal{V}}^{\mathrm{con}} \rightarrow-8 a^{2} \Delta\left(\xi_{\mu 5}\right) m_{\pi_{\mu 5}}^{2} \ln m_{\pi_{\mu 5}}^{2}-8 a^{2} \Delta\left(\xi_{\mu}\right) m_{\pi_{\mu}}^{2} \ln m_{\pi_{\mu}}^{2}-\sum_{B} a^{2} \Delta\left(\xi_{B}\right) m_{K_{B}}^{2} \ln m_{K_{B}}^{2}
$$

where $\Delta$ is given in eq. (7).

We can now put the results together to find the $\pi_{5}$ mass at one loop. Writing

$$
\left(m_{\pi_{5}}^{1-\text { loop }}\right)^{2}=m_{\pi_{5}}^{2}+\frac{1}{96 \pi^{2} f^{2}} \epsilon_{5}
$$

we have

$$
\epsilon_{5}=\Sigma_{2, \mathrm{KE}}^{\mathrm{con}}+\Sigma_{2, \mathrm{MASS}}^{\mathrm{con}}+\Sigma_{2, \mathcal{V}}^{\mathrm{con}}+\Sigma_{2}^{\text {disc }}-m_{\pi_{5}}^{2} \Sigma_{1}
$$

From eqs. (19), (20), (21), and (24) we then have

$$
\begin{aligned}
\epsilon_{5} \rightarrow m_{\pi_{5}}^{2}( & -3 m_{\pi_{5}}^{2} \ln m_{\pi_{5}}^{2}+12 m_{\pi_{\mu 5}}^{2} \ln m_{\pi_{\mu 5}}^{2}-18 m_{\pi_{\mu \nu}}^{2} \ln m_{\pi_{\mu \nu}}^{2} \\
+ & \left.\left.12 m_{\pi_{\mu}}^{2} \ln m_{\pi_{\mu}}^{2}-3 m_{\pi_{I}}^{2} \ln m_{\pi_{I}}^{2}\right)+\Sigma_{2}^{\text {disc }} \quad \text { [no sum on } \mu, \nu\right] .
\end{aligned}
$$

where the arrow, as usual, means that only the chiral logarithms are kept. Note that $\epsilon_{5}$ is proportional to $m_{\pi_{5}}^{2}$, as it must be by Goldstone's theorem. ( $\Sigma_{2}^{\text {disc }}$ already has a factor of $m_{\pi_{5}}^{2}$ in eq. (23).) The term $\sum_{B} m_{K_{B}}^{4} \ln \left(m_{K_{B}}^{2}\right)$ (eq. (20) $)$ combines with $-\sum_{B} a^{2} \Delta\left(\xi_{B}\right) m_{K_{B}}^{2} \ln \left(m_{K_{B}}^{2}\right)$ (eq. (24)) to cancel against $-m_{K_{5}}^{2} \sum_{B} m_{K_{B}}^{2} \ln \left(m_{K_{B}}^{2}\right)$ (eq. (21)). Here I have used 


$$
m_{K_{B}}^{2}=m_{K_{5}}^{2}+a^{2} \Delta\left(\xi_{B}\right)
$$

which follows from eqs. (15) and (7). Similarly, the terms $8 m_{\pi_{\mu 5}}^{4} \ln \left(m_{\pi_{\mu 5}}^{2}\right)$ and $8 m_{\pi_{\mu}}^{4} \ln \left(m_{\pi_{\mu}}^{2}\right)$ in eq. (21) combine with corresponding terms in eq. (24) to produce terms that have a factor of $m_{\pi_{5}}^{2}$. Indeed, eq. (24), the effect of the symmetry violating vertices, is completely determined by the requirement that the full answer go like $m_{\pi_{5}}^{2}$ (up to logs) in the chiral limit. This makes it clear, for example, that the $C_{i}$ coefficients in eq. (5) must enter eq. (24) only through the combinations $\Delta\left(\xi_{B}\right)$, and that the final answer can depend only on the various meson masses.

Terms of the form $m_{\pi_{5}}^{2} m_{K_{B}}^{2} \ln \left(m_{K_{B}}^{2}\right)$ also cancel between eqs. (19) and (21). This is "accidental" in the sense that it is not required by symmetry. It corresponds to the fact that there happen to be no $\ln \left(m_{K}^{2}\right)$ terms in the standard continuum $2+1$ flavor result 四. For similar quantities, such as $f_{\pi}$ or $\langle 0|\bar{u} u| 0\rangle$, the $\ln \left(m_{K}^{2}\right)$ terms will not cancel [4].

Finally, note that in the symmetry limit $\left(m_{\pi_{5}}=m_{\pi_{\mu 5}}=m_{\pi_{\mu \nu}}=m_{\pi_{\mu}}=m_{\pi_{I}}\right)$ the entire one-loop correction in eq. (27) is proportional to $\Sigma_{2}^{\text {disc }}$. This is also true of the standard result [4]; the reason will be explained in the next section.

\section{ONE-LOOP PION MASS FOR 2+1 DYNAMICAL FLAVORS}

To adjust the result in eq. (27) to the case of $2+1$ dynamical flavors, we need to identify the contribution from each of the possible quark flow diagrams shown in Fig. 1 . Many of the arguments used to identify the quark flows will be familiar from Ref. [7]. Once the contributions from virtual quark loops are determined, the adjustment to $2+1$ dynamical flavors is accomplished by multiplying every $\ell(i . e ., u, d)$ quark loop by $1 / 2$ and every $s$ quark loop by $1 / 4$.

First note that Figs. $4(\mathrm{~d}),(\mathrm{e})$, and (f) have disconnected internal meson propagators, and correspond to Fig. $\mathrm{Z}(\mathrm{b})$. Figure $6(\mathrm{~d})$ has a single $m_{0}^{2}$ insertion and therefore generates

the contribution in eq. (22). Figures [(e) and (f) represents the iteration of the $m_{0}^{2}$ vertex through the introduction of virtual quark loops. Together, Figs. ⿷(e) and (f) change eq. (22) 
to eq. (23).

To identify contributions from the remaining graphs in Fig. 1, we first study the possible 2 into 2 meson scattering diagrams in the quark flow language, Fig. 5. Letting $i, j, k, n=1,2, \cdots 8$ be quark flavor indices, Fig. 5(a) corresponds to a meson vertex of the form $\Phi_{i j} \Phi_{j i} \Phi_{k n} \Phi_{n k}$, where $\Phi$ is the meson field in eq. (9). Similarly Fig. 5(b) corresponds to $\Phi_{i j} \Phi_{j k} \Phi_{k n} \Phi_{n i}$. Now, consider the case where all four quark flavors $i, j, k, n$ are different. Then vertices generated by the kinetic energy or mass terms in eq. (11) cannot have the form $\Phi_{i j} \Phi_{j i} \Phi_{k n} \Phi_{n k}$ because they are formed only by a single trace of fields (and the diagonal matrix $\mathcal{M}$ ). Since Fig. $5($ a) cannot depend on the flavors of the quark lines to this order (except trivially through $\mathcal{M}$ ), this implies that Fig. $5($ a) is not generated at tree level by the kinetic or mass terms for any values of $i, j, k, n$.

The KS-flavor violating vertices behave differently. Because at least some of the $\xi_{B}$ explicitly entering eq. (5) must be off-diagonal, it is not hard to see that Fig. 5(a) can in fact be generated. Note, however, that since $\xi_{B}$ are diagonal under the vector $S U(2)$ that mixes the two KS fields, Fig. 5(a) still vanishes when $i, j$ are of $\ell$ type (upper $4 \times 4$ block) and $k, n$ are of $s$ type (lower $4 \times 4$ block).

Now consider Fig. $4(\mathrm{c})$. This graph is absent when the quarks in the virtual loops are of $s$ type because the needed vertex vanishes to this order. If we then choose $m_{s}=m_{\ell}$, it can make no difference whether a quark in a virtual loop is $\ell$ or $s$ type, so this graph must also vanish when the loop quarks are of $\ell$ type. A slight generalization of this argument shows that the graph again vanishes when there is one virtual quark of each type (i.e., a kaon loop).

Once we know that Fig. 4( (c) is absent, intermediate kaons could only be generated by

\footnotetext{
${ }^{8}$ Add a third $\left(s^{\prime}\right)$ KS quark field, plus a pseudoquark KS field $\tilde{s}^{\prime}$ of the same mass. This partially quenched theory is then equivalent to the original theory in the $\ell, s$ sector. The graph Fig. 田(c) then vanishes when there is one $s$ and one $s^{\prime}$ in the loop. Now choose $m_{s^{\prime}}=m_{\ell}$.
} 
Fig. $4(\mathrm{~b})$. Since the $K$ contribution to $\epsilon_{5}$ in fact vanishes to this order, graphs of type Fig. $4(\mathrm{~b})$ with an $s$ type quark in the virtual loop must cancel among themselves. Once more choosing $m_{s}=m_{\ell}$, this also implies that Fig. 田(b) cancels for a $\ell$ type virtual quark loop.

The surviving connected contribution to $\epsilon_{5}$ comes from Fig. 田(a). Furthermore, since the vertex in Fig. $4(\mathrm{a})$ is of the type in Fig. 5(a), which can only be generated by KS-flavor violating terms, such connected contributions must vanish in the symmetry limit. This is in fact the case, as was mentioned at the end of the last section. Because Fig. 1(a) contains valence quark lines only, these contributions do not change when we go from $4+4$ to $2+1$ dynamical flavors.

The only required adjustment is therefore to the disconnected contribution, $\Sigma_{2}^{\text {disc }}$. We need to divide every virtual quark loop contribution in Figs. 团(e) and (f) by 2 or 4, depending on whether it is $\ell$ or $s$ type, respectively. In eq. (23) this is easily accomplished by the replacements $\left(4 m_{0}^{2} / 3\right) /\left(q^{2}+m_{\pi_{I}}^{2}\right) \rightarrow\left(2 m_{0}^{2} / 3\right) /\left(q^{2}+m_{\pi_{I}}^{2}\right)$ and $\left(4 m_{0}^{2} / 3\right) /\left(q^{2}+m_{S_{I}}^{2}\right) \rightarrow$ $\left(m_{0}^{2} / 3\right) /\left(q^{2}+m_{S_{I}}^{2}\right)$ in the denominator. In other words, we have in the $2+1$ case:

$$
\Sigma_{2}^{\text {disc }}=3 m_{\pi_{5}}^{2} \int \frac{d^{4} q}{\pi^{2}} \frac{4 m_{0}^{2} / 3}{\left(q^{2}+m_{\pi_{I}}^{2}\right)^{2}\left(1+\frac{2 m_{0}^{2} / 3}{q^{2}+m_{\pi_{I}}^{2}}+\frac{m_{0}^{2} / 3}{q^{2}+m_{S_{I}}^{2}}\right)} .
$$

We can now take the limit $m_{0}^{2} \rightarrow \infty$ and put eq. (29) in the form

$$
\Sigma_{2}^{\text {disc }}=m_{\pi_{5}}^{2} \int \frac{d^{4} q}{\pi^{2}}\left(\frac{6}{\left(q^{2}+m_{\pi_{I}}^{2}\right)}-\frac{2}{\left(q^{2}+m_{\eta_{I}}^{2}\right)}\right),
$$

where $m_{\eta_{I}}^{2} \equiv\left(2 m_{S_{I}}^{2}+m_{\pi_{I}}^{2}\right) / 3$. Equation (17) then gives

$$
\Sigma_{2}^{\text {disc }} \rightarrow m_{\pi_{5}}^{2}\left(6 m_{\pi_{I}}^{2} \ln m_{\pi_{I}}^{2}-2 m_{\eta_{I}}^{2} \ln m_{\eta_{I}}^{2}\right)
$$

Inserting this into eq. (27), we get the $2+1$ result for the chiral logarithms

$$
\begin{aligned}
\epsilon_{5} \rightarrow m_{\pi_{5}}^{2}( & -3 m_{\pi_{5}}^{2} \ln m_{\pi_{5}}^{2}+12 m_{\pi_{\mu 5}}^{2} \ln m_{\pi_{\mu 5}}^{2}-18 m_{\pi_{\mu \nu}}^{2} \ln m_{\pi_{\mu \nu}}^{2} \\
+ & \left.\left.12 m_{\pi_{\mu}}^{2} \ln m_{\pi_{\mu}}^{2}+3 m_{\pi_{I}}^{2} \ln m_{\pi_{I}}^{2}-2 m_{\eta_{I}}^{2} \ln m_{\eta_{I}}^{2}\right) \quad \text { [no sum on } \mu, \nu\right] .
\end{aligned}
$$

In the KS-symmetry limit, this gives the standard result [4]. As discussed above, the result comes entirely from $\Sigma_{2}^{\text {disc }}$ in this limit. 
What about the analytic terms? These can in general come from a quite complicated set of operators in the chiral theory: standard $\mathcal{O}\left(m^{2}\right)\left(" p^{4}\right.$ ") operators 4 , $\mathcal{O}\left(m a^{2}\right)$ (e.g., $\left.\mathcal{V} \operatorname{tr}\left(\mathcal{M} \Sigma+\mathcal{M} \Sigma^{\dagger}\right)\right)$ or $\mathcal{O}\left(a^{4}\right)\left(e . g ., \mathcal{V}^{2}\right)$ chiral operators generated by the same terms in the continuum effective action that led to eq. (11), and entirely new chiral operators of $\mathcal{O}\left(m a^{2}\right)$ and $\mathcal{O}\left(a^{4}\right)$ coming for example from terms in the continuum action that Lee and Sharpe [6] call $S_{6}^{\mathrm{FF}(\mathrm{B})}$, which break the symmetries of eq. (11) down to the lattice symmetries. However, as a function of quark mass, the analytic terms in $\left(m_{\pi_{5}}^{1-l o o p}\right)^{2}$ of the relevant order can only be proportional to $m_{\ell}, m_{\ell}^{2}$ or $m_{\ell} m_{s}$, since they must vanish as $m_{\ell} \rightarrow 0$. This means that terms of $\mathcal{O}\left(a^{4}\right)$ in the joint $m, a^{2}$ expansion cannot enter.

Putting eq. (32) in eq. (25), adding in the analytic terms just discussed, and writing the overall $m_{\pi_{5}}^{2}$ as $2 \mu m_{\ell}$ (eqs. (15) and (7)), I arrive at the final result in the $2+1$ case (no sum on $\mu, \nu)$ :

$$
\begin{aligned}
& \left(m_{\pi_{5}}^{1-\text { loop }}\right)^{2} / m_{\ell}=2 \mu\left\{1+\frac{1}{16 \pi^{2} f^{2}}\left(-\frac{1}{2} m_{\pi_{5}}^{2} \ln \frac{m_{\pi_{5}}^{2}}{\Lambda^{2}}+2 m_{\pi_{\mu 5}}^{2} \ln \frac{m_{\pi_{\mu 5}}^{2}}{\Lambda^{2}}-3 m_{\pi_{\mu \nu}}^{2} \ln \frac{m_{\pi_{\mu \nu}}^{2}}{\Lambda^{2}}\right.\right. \\
& \left.\quad+2 m_{\pi_{\mu}}^{2} \ln \frac{m_{\pi_{\mu}}^{2}}{\Lambda^{2}}+\frac{1}{2} m_{\pi_{I}}^{2} \ln \frac{m_{\pi_{I}}^{2}}{\Lambda^{2}}-\frac{1}{3} m_{\eta_{I}}^{2} \ln \frac{m_{\eta_{I}}^{2}}{\Lambda^{2}}+2 m_{\ell} K_{3}+\left(2 m_{\ell}+m_{s}\right) K_{4}^{\prime}+a^{2} C\right\}
\end{aligned}
$$

where $\Lambda$ is the chiral scale and $K_{3}, K_{4}^{\prime}$, and $C$ are independent of $m$ and $a$ to this order. The term $a^{2} C$ may alternatively be considered as a discretization correction to $\mu$. $K_{4}^{\prime}$ is related to the parameter $K_{4}$ of Ref. [4] by

$$
K_{4}=\left(2 m_{\ell}+m_{s}\right) K_{4}^{\prime}
$$

In the KS-symmetry limit eq. (33) reduces to the result in [4].

Since the term $a^{2} C$ is what absorbs mass-independent cutoff dependence in eq. (33), we can make a rough estimate of its size by computing the change of the logarithms when $\Lambda$ changes between two reasonable values, say 0.5 and $1.0 \mathrm{GeV}$. For MILC simulations at $a \approx 0.13 \mathrm{fm}$, for which $\Delta_{\max } \approx(400 \mathrm{MeV})^{2}$ (see discussion before eq. (1)), I find $a^{2} C \sim 0.05$. Discretization corrections to $K_{3}$ and $K_{4}^{\prime}$ come from higher, $\mathcal{O}\left(m^{2} a^{2}\right)$, contributions not considered in eq. (33). I expect that such corrections will be significant because they can 
be generated by the same operators in the effective QCD Lagrangian that produced the KS-symmetry breaking potential, $\mathcal{V}$.

\section{ONE-LOOP PION MASS IN THE QUENCHED CASE}

Given the understanding of the quark flows in Fig. I developed in the previous section, the result in the quenched case is easy to write down. $\Sigma_{2}^{\text {disc }}$ in eq. (27) changes to the quenched version, $\Sigma_{2}^{\text {disc,quench }}$, which corresponds to Fig. $4($ d) only. The other terms in eq. (27), which arise from Fig. 1(a), are unaffected. We thus have, in the quenched case

$$
\begin{aligned}
& \epsilon_{5} \rightarrow m_{\pi_{5}}^{2}(-3 m_{\pi_{5}}^{2} \ln m_{\pi_{5}}^{2}+12 m_{\pi_{\mu 5}}^{2} \ln m_{\pi_{\mu 5}}^{2}-18 m_{\pi_{\mu \nu}}^{2} \ln m_{\pi_{\mu \nu}}^{2} \\
&+ \\
&\left.\left.+12 m_{\pi_{\mu}}^{2} \ln m_{\pi_{\mu}}^{2}-3 m_{\pi_{I}}^{2} \ln m_{\pi_{I}}^{2}\right)+\Sigma_{2}^{\text {disc,quench }} \quad \text { [no sum on } \mu, \nu\right],
\end{aligned}
$$

with $\Sigma_{2}^{\text {disc,quench }}$ given by eq. (22).

Since $m_{0}^{2}$ is not to be taken to infinity in the quenched case, it is necessary to consider other $\phi_{I}$ dependence in the Lee-Sharpe Lagrangian, [? eq. (4). In Ref. [8] it was shown that it is sufficient to add in a contribution to the $\phi_{I}$ kinetic energy: $(2 \alpha / 3)\left(\partial_{\mu} \phi_{I}\right)^{2}$. This merely changes $m_{0}^{2}$ in eq. (22) to $m_{0}^{2}+\alpha q^{2}$.

The non-analytic terms in $\Sigma_{2}^{\text {disc,quench }}$ may be extracted using

$$
\begin{aligned}
& \mathcal{I}_{3} \equiv \int \frac{d^{4} q}{(2 \pi)^{4}} \frac{1}{\left(q^{2}+m^{2}\right)^{2}}=\frac{-1}{2 m} \frac{\partial}{\partial m} \mathcal{I}_{1} \rightarrow-\frac{1}{16 \pi^{2}} \ln m^{2} \\
& \mathcal{I}_{4} \equiv \int \frac{d^{4} q}{(2 \pi)^{4}} \frac{q^{2}}{\left(q^{2}+m^{2}\right)^{2}}=\frac{-1}{2 m} \frac{\partial}{\partial m} \mathcal{I}_{2}=\mathcal{I}_{1}-m^{2} \mathcal{I}_{3} \rightarrow \frac{1}{16 \pi^{2}} 2 m^{2} \ln m^{2},
\end{aligned}
$$

where $\mathcal{I}_{1}$ and $\mathcal{I}_{2}$ are defined in eqs. (17) and (18).

Putting the logarithms in $\Sigma_{2}^{\text {disc,quench }}$ together with eq. (35), and using eq. (25), gives the final quenched answer:

\footnotetext{
${ }^{9}$ In the quenched case, there is no point in going to the $4+4$ Lagrangian, eq. (11), since $s$ type quarks do not couple to the pion.
} 


$$
\begin{aligned}
& \left(m_{\pi_{5}}^{1 \text { loop }}\right)^{2} / m_{\ell}=2 \mu\left\{1+\frac{1}{16 \pi^{2} f^{2}}\left(-\frac{1}{2} m_{\pi_{5}}^{2} \ln \frac{m_{\pi_{5}}^{2}}{\Lambda^{2}}+2 m_{\pi_{\mu 5}}^{2} \ln \frac{m_{\pi_{\mu 5}}^{2}}{\Lambda^{2}}-3 m_{\pi_{\mu \nu}}^{2} \ln \frac{m_{\pi_{\mu \nu}}^{2}}{\Lambda^{2}}\right.\right. \\
& \left.\left.\quad+2 m_{\pi_{\mu}}^{2} \ln \frac{m_{\pi_{\mu}}^{2}}{\Lambda^{2}}-\frac{1}{2} m_{\pi_{I}}^{2} \ln \frac{m_{\pi_{I}}^{2}}{\Lambda^{2}}-\frac{2}{3} m_{0}^{2} \ln \frac{m_{\pi_{I}}^{2}}{\Lambda^{2}}+\frac{4}{3} \alpha m_{\pi_{I}}^{2} \ln \frac{m_{\pi_{I}}^{2}}{\Lambda^{2}}\right)+2 m_{\ell} K_{3}+a^{2} C\right\}
\end{aligned}
$$

where of course the parameters $\mu, K_{3}$ and $C$ may be different from those in eq. (33). I keep the term multiplying $\alpha$ separate from the other $\ln m_{\pi_{I}}^{2}$ term, since they arise from different integrals and therefore will have different finite volume corrections. Analytic terms depending on $m_{0}^{2}$ or $\alpha$ are not included since they can be absorbed in $\mu$ and $\Lambda$. Note that the parameter $K_{4}^{\prime}$ does not appear here because it multiplies $m_{s}$, which cannot enter in the quenched pion mass.

In the symmetric limit, eq. (38) agrees with results in [8] or [7]. As usual, the power counting in the quenched case is somewhat problematical; one must assume that $m_{0}^{2}$ and $\alpha$ are in some sense small to stop at one loop [8,7].

\section{FINITE VOLUME CORRECTIONS}

The technique for computing the finite volume corrections to the results of Secs. $\square$ and $\mathrm{V}$ is standard 13,14. However, I will provide enough detail here to allow the reader to include the finite volume corrections numerically in fits to eqs. (33) and (38). I assume that the Euclidean time dimension, $T$, is large enough that it may be taken infinite, and only corrections from the finite spatial dimensions (length $L$ ) need be considered. The generalization to the case where $T$ is also finite will be obvious.

The results in finite volume come simply from replacing the integrals $\mathcal{I}_{i}(i=1, \ldots, 4)$ in

eqs. (17), (18), (36) and (37) by their finite volume counterparts $\mathcal{I}_{i}^{(L)}$. We have, for example,

$$
\begin{aligned}
\mathcal{I}_{1}^{(L)} & \equiv \frac{1}{L^{3}} \sum_{\vec{n}} \int \frac{d q_{0}}{(2 \pi)} \frac{1}{q_{0}^{2}+\left(\vec{q}_{n}\right)^{2}+m^{2}} \\
\vec{q}_{n} & =2 \pi \vec{n} / L ; \quad \vec{n}=\left(n_{x}, n_{y}, n_{z}\right)
\end{aligned}
$$

where $n_{x}, n_{y}$, and $n_{z}$ are integers, and I am assuming periodic boundary conditions. $\mathcal{I}_{2}^{(L)}$ 
$\mathcal{I}_{3}^{(L)}$ and $\mathcal{I}_{4}^{(L)}$ are defined analogously.

Since the integrals of interest are divergent, it is preferable to work with the differences $\mathcal{I}_{i}^{(L)}-\mathcal{I}_{i}$, which are finite as long as care is taken in introducing a cutoff. Examples of acceptable regulators include multiplying all integrands/summands by $\left(\Lambda^{2} /\left(q^{2}+\Lambda^{2}\right)\right)^{k}$ (where $k$ is a large enough power to render the integrals convergent) [15, point-splitting the corresponding position-space propagators, and defining the theory on a lattice.

Assuming the integrals/sums have been regulated appropriately, I define the dimensionless finite volume corrections $\delta_{i}$ by

$$
\begin{aligned}
& \mathcal{I}_{1}^{(L)}-\mathcal{I}_{1}=\frac{m^{2}}{16 \pi^{2}} \delta_{1}(m L) \\
& \mathcal{I}_{2}^{(L)}-\mathcal{I}_{2}=\frac{m^{4}}{16 \pi^{2}} \delta_{2}(m L) \\
& \mathcal{I}_{3}^{(L)}-\mathcal{I}_{3}=\frac{1}{16 \pi^{2}} \delta_{3}(m L) \\
& \mathcal{I}_{4}^{(L)}-\mathcal{I}_{4}=\frac{m^{2}}{16 \pi^{2}} \delta_{4}(m L)
\end{aligned}
$$

The correction $\delta_{1}$ can easily be written in terms of the Euclidean position space propagator, defined in infinite volume by

$$
G(x) \equiv \int \frac{d^{4} q}{(2 \pi)^{4}} \frac{e^{-i q \cdot x}}{q^{2}+m^{2}}=\frac{m}{4 \pi^{2}|x|} K_{1}(m|x|)
$$

where $K_{1}$ is a Bessel function of imaginary argument. The corresponding finite volume propagator, is given by

$$
G^{(L)}(x) \equiv \frac{1}{L^{3}} \sum_{\vec{n}} \int \frac{d q_{0}}{(2 \pi)} \frac{e^{-i q \cdot x}}{q_{0}^{2}+\left(\vec{q}_{n}\right)^{2}+m^{2}}=G(x)+\sum_{\vec{n} \neq 0} G(x+L \vec{n})
$$

with $q=\left(q_{0}, \vec{q}_{n}\right)$. The last equality follows from the Poisson resummation formula, or simply by noting that it is the unique solution of the corresponding differential equation with the correct (periodic) boundary conditions. Subtracting eqs. (46) and (45), setting $x=0$, and putting the result into eq. (41) using eqs. (39) and (17) gives

$$
\delta_{1}(m L)=\frac{4}{m L} \sum_{\vec{n} \neq 0} \frac{K_{1}(|\vec{n}| m L)}{|\vec{n}|} .
$$


For practical values of $m L, \delta_{1}$ can be readily computed to machine precision with this formula. (One is limited of course by the accuracy to which $K_{1}$ is evaluated.) An alternative approach, inspired by Ref. [16], is to compute the difference of the (regulated) sum and integral directly in momentum space, treating the sum as an integral by rounding $L \vec{q} /(2 \pi)$ to the nearest $\vec{n}$ and using a standard numerical integration program [17]. With a reasonable regulator (e.g., the one from [15], mentioned above), this method gives results consistent with eq. (47), but it is much slower.

The other finite volume corrections follow from the relations among the integrals given in eqs. (18), (36) and (37) and the standard recursion relation $K_{1}^{\prime}(z)=-K_{1}(z) / z-K_{0}(z)$, where prime implies differentiation. I find

$$
\begin{aligned}
& \delta_{2}(m L)=-\delta_{1}(m L) \\
& \delta_{3}(m L)=-\delta_{1}(m L)-\frac{m L}{2} \delta_{1}^{\prime}(m L)=2 \sum_{\vec{n} \neq 0} K_{0}(|\vec{n}| m L) \\
& \delta_{4}(m L)=2 \delta_{1}(m L)+\frac{m L}{2} \delta_{1}^{\prime}(m L)=\delta_{1}(m L)-\delta_{3}(m L)
\end{aligned}
$$

In deriving eq. (48), one must be careful to regulate so that the difference between the sum and the integral of unity vanishes in the limit of infinite regulator. The regulator in 15 works well, for example.

Large $m L$ asymptotic expressions for the $\delta_{i}$ are obtained by including only the 6 nearest neighbors in the sums in eqs. (47) and (49) and using the only leading terms in $K_{1}$ and $K_{0}$. I find

$$
\begin{aligned}
& \delta_{1}(m L)=-\delta_{2}(m L) \sim \frac{12 \sqrt{2 \pi}}{(m L)^{\frac{3}{2}}} e^{-m L} \\
& \delta_{3}(m L) \sim-\delta_{4}(m L) \sim 6 \sqrt{\frac{2 \pi}{m L}} e^{-m L} .
\end{aligned}
$$

While these expressions are useful for estimating the size of finite size effects, the leading corrections to eqs. (51) and (52) have just one higher power of $1 /(m L)$. In the quantitative applications I have in mind [11], for which $m L$ is in the range 3 to 9 , the full expressions (eqs. (47), (48), (49) and (50) ) - or at least the full $K_{1}$ and $K_{0}$ from the nearest neighbors 
— will be needed. It is important to note, however, that for very small quark masses, $m L \lesssim 1$, eqs. (47), (48), (49) and (50) are not applicable, no matter how many terms are included in the sums. In that limit, the zero-mode (the spatially independent component of the pion field) must be treated exactly [14].

Comparing eqs. (41)-(44) with eqs. (17), (18), (36) and (37) then gives the rules for including finite volume corrections. In the $2+1$ case, one needs merely to make the replacement

$$
\ln \frac{m^{2}}{\Lambda^{2}} \rightarrow \ln \frac{m^{2}}{\Lambda^{2}}+\delta_{1}(m L)
$$

for every logarithm in eq. (33). It is interesting to note that when $m_{\ell} \rightarrow 0$ (with $L$ and the symmetry violating parameters, $C_{i}$, fixed), the leading finite size correction to eq. (33) is negative, due to the $-\frac{1}{2} m_{\pi_{5}}^{2} \ln m_{\pi_{5}}^{2}$ term. In the standard case, the leading correction is positive, due to the positive sign of $m_{\pi}^{2} \ln m_{\pi}^{2}$.

In the quenched approximation, the replacements in eq. (38) are:

$$
\begin{aligned}
m_{0}^{2} \ln \frac{m_{\pi_{I}}^{2}}{\Lambda^{2}} & \rightarrow m_{0}^{2} \ln \frac{m_{\pi_{I}}^{2}}{\Lambda^{2}}-m_{0}^{2} \delta_{3}\left(m_{\pi_{I}} L\right) \\
\alpha \ln \frac{m_{\pi_{I}}^{2}}{\Lambda^{2}} & \rightarrow \alpha \ln \frac{m_{\pi_{I}}^{2}}{\Lambda^{2}}+\frac{1}{2} \alpha \delta_{4}\left(m_{\pi_{I}} L\right) \\
\ln \frac{m^{2}}{\Lambda^{2}} & \rightarrow \ln \frac{m^{2}}{\Lambda^{2}}+\delta_{1}(m L) \quad \text { [all other logarithms] }
\end{aligned}
$$

\section{REMARKS AND CONCLUSIONS}

My main results are given for the $2+1$ flavor case in eq. (33) and for the quenched case in eq. (38). Finite volume corrections to these formulas appear in eqs. (53) and (54), respectively, with the values of $\delta_{i}$ given in eqs. (47) to (50).

I have computed only $m_{\pi_{5}}^{2}$, but a generalization of this calculation to $m_{K_{5}}^{2}$ is straightforward and is in progress [18]. Slightly more complicated, but also on the list of things

to compute [18] are $f_{\pi}, f_{K},\langle\bar{\Psi} \Psi\rangle$, and the non-Goldstone $\pi$ and $K$ masses. These quantities do not vanish in the chiral limit, so there will be fewer short-cuts available. A further 
complication for a quantity like $f_{\pi}$ is that there is no accidental cancellation of diagrams corresponding to Fig. $4(\mathrm{~b})$. An additional adjustment for the number of flavors in virtual quark loops will therefore be required. However, the adjustment is not difficult, since $\ln m_{K}^{2}$ contributions come uniquely from this diagram. To generate the $2+1$ case, one will merely have to: (1) multiply the $\ln m_{K}^{2}$ terms by $1 / 4$ to count the $s$ quarks in the loop correctly, and (2) subtract half the (original) $\ln m_{K}^{2}$ terms, after putting $m_{s} \rightarrow m_{\ell}$, to count $\ell$ quarks

in the loop correctly. For quantities such as $f_{K}$ or $m_{K}^{2}$, where there will be valence $\ln m_{K}^{2}$ contributions, the $\ln m_{\pi}^{2}$ and $\ln m_{S}^{2}$ terms (from the connected internal propagator) can be used to make the adjustment.

Preliminary fits of eq. (33) to MILC $2+1$ data are encouraging: the fits give good confidence levels for reasonable ranges of quark mass [11]. The contrast with the standard fits that do not take into account KS flavor breaking [3] is especially striking. I emphasize here that the new fits have the same number of free parameters as the standard ones. This is because the various pion splittings are not left free in eq. (33) but are determined first by the data for the masses of all the pions.

In the quenched case, one generally treats $m_{0}$ (or equivalently $\delta \equiv m_{0}^{2} /\left(24 \pi^{2} f^{2}\right)$ ) as a free parameter. As such, decent fits can apparently be performed whether or not one takes flavor breaking into account. However, the values of $\delta$ obtained seem to be considerably closer to the real world values $(\delta \approx 0.18)$ when flavor breaking effects are included. In both the $2+1$ and quenched cases, we are collecting additional lattice data at small quark mass [11], which should significantly increase the discrimination between the symmetric and flavor-breaking fits.

\section{ACKNOWLEDGMENTS}

I am grateful to M. Golterman, P. Hasenfratz, K. Orginos, S. Sharpe, and D. Toussaint, as well as my other colleagues in MILC, for very useful discussions. M. Golterman, S. Sharpe, and D. Toussaint also provided helpful comments on an earlier draft of this manuscript. Some 
of this work was completed during the program "Lattice QCD and Hadron Phenomenology" at the Institute for Nuclear Theory, University of Washington (Fall, 2001). I thank the INT staff and organizers M. Golterman and S. Sharpe for a productive stay.

[1] The MILC Collaboration: C. Bernard et al., Phys. Rev. D 58, 014503 (1998); The MILC Collaboration: T. Blum et al., Phys. Rev. D 55, 1133 (1997); G.P. Lepage, Nucl. Phys. (Proc. Suppl.) 60A, 267 (1998); K. Orginos and D. Toussaint, Phys. Rev. D 59, 014501 (1999); Nucl. Phys. B (Proc. Suppl.) 73, 909 (1999); J.F. Lagäe and D.K. Sinclair, Nucl. Phys. (Proc. Suppl.) 63, 892 (1998); Phys. Rev. D 59, 014511 (1999); K. Orginos, D. Toussaint and R.L. Sugar, Phys. Rev. D 60, 054503 (1999); Nucl. Phys. B (Proc. Suppl.) 83-84, 878 (2000); G.P. Lepage, Phys. Rev. D 59, 074502 (1999).

[2] The MILC collaboration: C. Bernard et al., Phys. Rev. D 61, 111502(R) (2000); The MILC Collaboration: C. Bernard et al., Phys. Rev. D 62, 034503 (2000).

[3] The MILC Collaboration: C. Bernard et al., Phys. Rev. D 64, 054506 (2001).

[4] J. Gasser and H. Leutwyler, Nucl. Phys. B250, 465 (1985).

[5] It was K. Orginos who first suggested to me that KS-flavor violation might be directly responsible for the difficulties with chiral fits in Ref. 3 and that a one-loop calculation using the Lagrangian of Ref. [6] might be necessary.

[6] W. Lee and S.R. Sharpe, Phys. Rev. D60, 114503 (1999).

[7] S.R. Sharpe, Nucl. Phys. B (Proc. Suppl.) 17, 146 (1990) and Phys. Rev. D46, 3146 (1992).

[8] C. Bernard and M. Golterman, Phys. Rev. D46, 853 (1992).

[9] C. Bernard and M. Golterman, Phys. Rev. D49, 486 (1994).

[10] P. Hasenfratz points out (private communication) that one should always be able to write 
down a standard (à la Ref. 㧄) 2+1 flavor chiral theory, and add arbitrary symmetry violating terms to represent $\mathcal{O}\left(a^{2}\right)$ effects. But without a corresponding quark level Lagrangian, I see no way to organize and limit these terms to get out useful information.

[11] The MILC collaboration, in preparation.

[12] S.R. Sharpe and N. Shoresh, hep-lat/0108003.

[13] J. Gasser and H. Leutwyler, Phys. Lett. 184B (1987) 83, Phys. Lett. 188B (1987) 477, and Nucl. Phys. B307 (1988) 763; H. Leutwyler, Nucl. Phys. B (Proc. Suppl.) 4 (1988) 248 and Phys. Lett. 189B (1987) 197; H. Neuberger, Nucl. Phys. B300 (1988) 180; P. Hasenfratz and H. Leutwyler, Nucl. Phys. B343 (1990) 241. My approach here is most closely related to that of [7] and [8].

[14] J. Gasser and H. Leutwyler, Phys. Lett. 184B (1987) 83, Phys. Lett. 188B (1987) 477.

[15] M. Golterman and K.C. Leung, Phys. Rev. D56 (1997) 2950.

[16] M. Nobes, H. Trottier, G.P. Lepage, and Q. Mason, presented at the International Symposium, Lattice 2001, Berlin, August 19-24, 2001, to be published in Nucl. Phys. B (Proc. Suppl.), hep-lat/0110051.

[17] G. P. Lepage, "Vegas: An Adaptive Multidimensional Integration Program," CLNS-80/447.

[18] C. Aubin and C. Bernard, work in progress. 


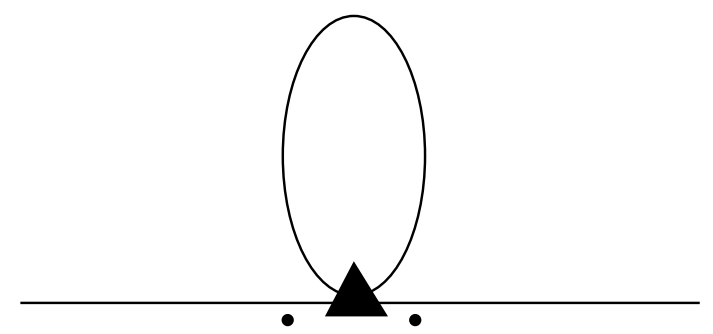

(a)

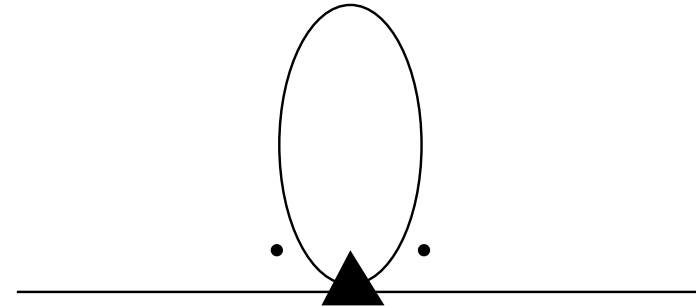

(b)

FIG. 1. Chiral perturbation theory graphs contributing to the pion propagator from kinetic energy vertex (solid square). The external lines are Goldstone pions, i.e., $\pi_{5}$. The dots represent the derivatives in the vertex. In (a) they act on the external lines; in (b), the internal.

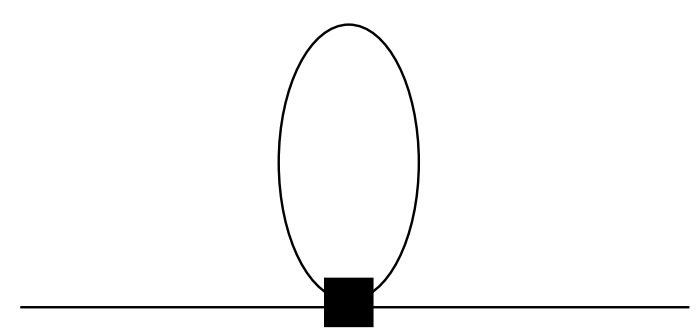

(a)

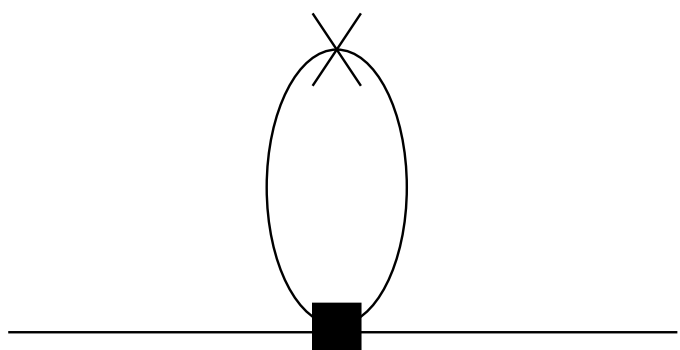

(b)

FIG. 2. Same as Fig. 1, but from the mass vertex (solid triangle). The internal propagator in (a) is the connected propagator only (no $m_{0}^{2}$ insertions), even when it is neutral $\left(\pi_{I}\right)$. All disconnected contributions are in (b); i.e., the cross represents one or more insertions of the $m_{0}^{2}$ vertex. 


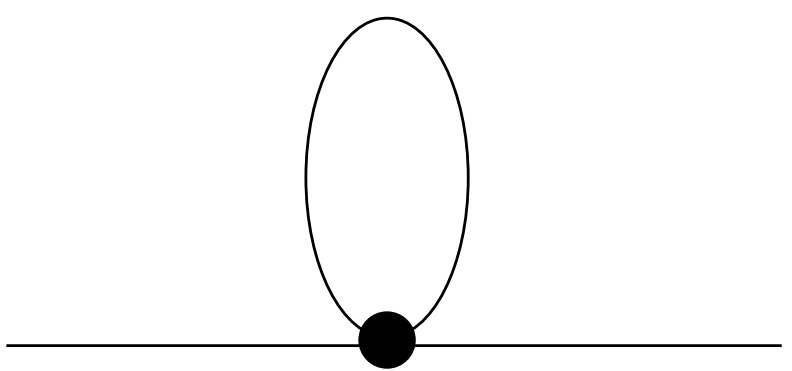

FIG. 3. Same as Fig. 1, but from the flavor breaking vertex $\mathcal{V}$ (solid circle). 


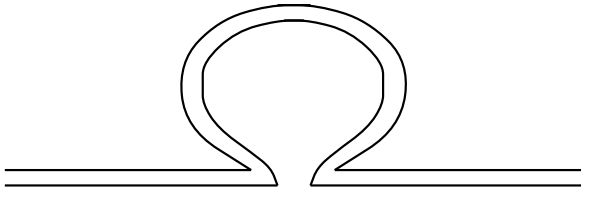

(a)

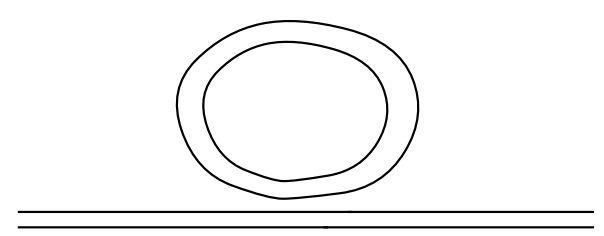

(c)

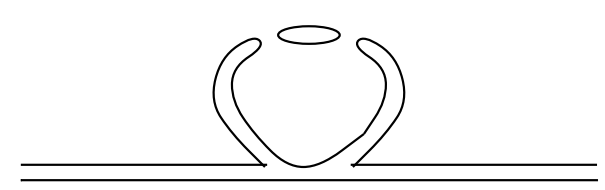

(e)

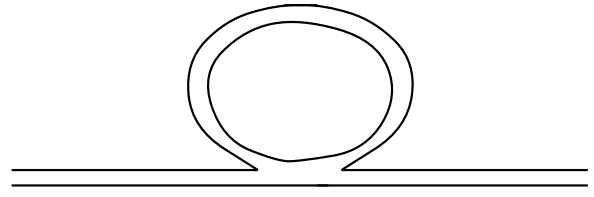

(b)

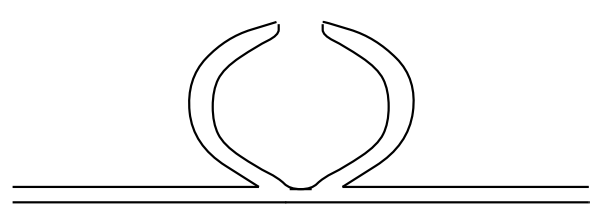

(d)

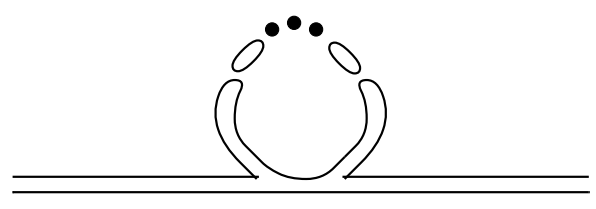

(f)

FIG. 4 .

Quark flow diagrams that could contribute to the (flavor non-singlet) pion propagator at one loop in the chiral expansion. Diagrams (d), (e) and (f) are disconnected graphs that correspond to figure Fig. 2(b); (f) represents the sum over two or more intermediate virtual quark loops. Note that (d), (e) and (f) each have a second version, in which the two sides of the meson loop come from different valence lines. For the purposes of this paper, one may consider the diagrams shown to be generic, subsuming both versions. Diagrams with " $\Phi_{0}$ vertices," such as Fig. 3(i) of Ref. [7], are not relevant: either because they are eliminated by the $m_{0}^{2} \rightarrow \infty$ limit (the $2+1$ case), or because they have virtual quark loops (the quenched case). Diagrams with odd numbers of $\Phi_{0}$ lines at a vertex, such as Fig. 3(f) of Ref. [7], can be eliminated by a field redefinition [ [ $]$ ] 


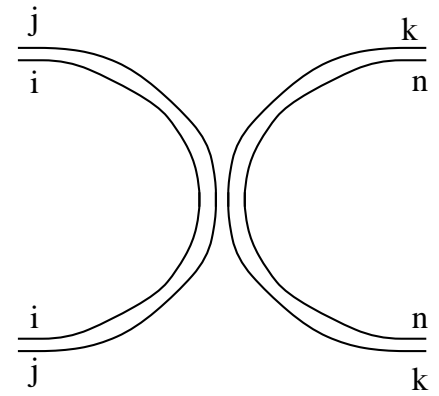

(a)

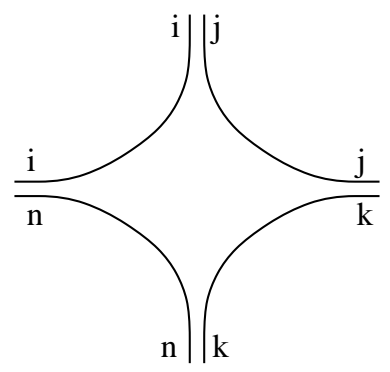

(b)

FIG. 5. Quark flow diagrams corresponding to 2 into 2 meson scattering at tree level in the chiral expansion. $\mathrm{i}, \mathrm{j}, \mathrm{k}, \mathrm{n}$ are flavor indices. 